\title{
HOMOTOPY ALGORITHM FOR BOX-CONSTRAINED LASSO AND ITS CONVERGENCE
}

\author{
Xijun Liang ${ }^{1}$, Yongxiang Wang $^{2}$ \\ ${ }^{1,2}$ College of Science \\ China University of Petroleum \\ Qingdao, P.R. CHINA
}

\begin{abstract}
The least absolute shrinkage and selection operator (Lasso) has received extensive attentions when sparse solutions are required. The homotopy method has become a significant approach for Lasso models because it can produce an entire regularization path. Although convergence has been studied on some special design matrices, the convergence analysis of the general homotopy method remains a problem.

In this work, we present a homotopy method for the generalized Lasso model with box constraints. We propose mild convergence assumptions and prove that the algorithm terminates at an optimal solution under convergence assumptions.
\end{abstract}

AMS Subject Classification: 90C25, 90C20

Key Words: LASSO, homotopy, box constraints, regularization path

\section{Introduction}

The Lasso model [1] and its variations have been widely studied, and applied in model selection, compressive sensing and logistic regression. Lasso is closely related to Dantzig selector. They have been compared and discussed theoretically and numerically, e.g., [2]. It was emphasized in [2] that computing Dantzig selector or Lasso for a single value of the penalty parameter does not work in practice, and the entire solution path is needed to select a meaningful model with good predictive performance.

Received: September 13, 2016

Revised: $\quad$ November 14, 2016

Published: $\quad$ February 1, 2017

$\S_{\text {Correspondence author }}$ (c) 2017 Academic Publications, Ltd. url: www.acadpubl.eu 
The homotopy method $[3,4]$ can generate the entire solution path in one run and it has shown encouraging numerical results [5]. While numerical results of existing homotopy algorithms are promising, limit attention has been given to the convergence analysis. For special design matrices, D. L. Donoho and Y. Tsaig [6] proved that the classical homotopy algorithm has $k$-step solution property. However, the assumed condition does not hold on many practical datasets and the sequence of regularization parameters may not strictly decrease. If recursion occurs, the algorithm fails to find an optimal solution.

In this work, we consider a general Lasso model for recommender systems [7], in which box constraints are added to avoid a predominant variable. We propose a generalized homotopy algorithm to seek for sparse solutions. Under convergence assumptions, we prove that the proposed homotopy algorithm terminates at an optimal solution. These assumptions, especially the nondegeneration index assumption, ensures that the degeneration case will not occur and the sequence of regularization parameters strictly decreases.

\section{The homotopy Algorithm for the Box-Constrained Lasso}

The box-constrained Lasso model can be recast as follows:

$$
\begin{array}{cl}
\min _{x} & \frac{1}{2}\|A x-y\|_{2}^{2}+\lambda\|x\|_{1} \\
\text { s.t. } & \|W x\|_{\infty} \leq \kappa .
\end{array}
$$

where design matrix $A:=\left[\begin{array}{lll}a_{1} & \ldots & a_{n}\end{array}\right] \in \mathbb{R}^{m, n}, m \leq n, y \in \mathbb{R}^{m}$, regularization parameter $\lambda>0$ and $\kappa>0$ are pre-determined parameters, $W$ is a diagonal matrix $\operatorname{Diag}\left(w_{1}, \ldots, w_{n}\right), w_{i} \geq 0$. Model (1) degenerates to the classical Lasso when $W=0$.

We generalize the classical homotopy algorithm [3, 4] for solving model (1). As model (1) is a convex, a vector $x$ is optimal if and only if there exists $u \in \mathbb{R}$ such that $(x, u)$ satisfies the KKT conditions:

$$
\left\{\begin{array}{l}
0 \in A^{T}(A x-y)+\lambda \cdot \partial\|x\|_{1}+u \cdot \partial\|W x\|_{\infty} \\
\|W x\|_{\infty} \leq \kappa, \\
u \geq 0, \quad u\left(\|W x\|_{\infty}-\kappa\right)=0 .
\end{array}\right.
$$

Denote $I=\left\{i \mid x_{i} \neq 0\right\}$ the active index set. Let

$$
I_{1}=\left\{i|| w_{i} x_{i} \mid=\kappa\right\}, \quad I_{2}=I \backslash I_{1}, \quad I_{3}=\{1, \ldots, n\} \backslash I .
$$

For any index set $J$, denote $A_{J}$ the submatrix consisting of the columns with the indices in $J$, and $x(J)$ the vector consisting of the elements of the 
vector $x$ with the indices in $J$. Let $\operatorname{co}\{\cdot\}$ be convex hull of a give set. Condition (2) can be written as

$$
\left\{\begin{array}{l}
-A_{I_{1}}^{T}(A x-y) \in \lambda \cdot \operatorname{sgn}\left(x\left(I_{1}\right)\right)+u \cdot \operatorname{co}_{i \in I_{1}}\left\{\operatorname{sgn}\left(x_{i}\right) w_{i} e_{i}\left(I_{1}\right)\right\} \\
-A_{I_{2}}^{T}(A x-y)=\lambda \cdot \operatorname{sgn}\left(x\left(I_{2}\right)\right) \\
\left|A_{I_{3}}^{T}(A x-y)\right| \leq \lambda
\end{array}\right.
$$

where $e_{i}$ is a vector of all 0 's except 1 at the $i$-th position.

Let $c=-A^{T}(A x-y)$. It follows by (6), (7) that $\operatorname{sgn}\left(c^{k}\left(I_{1}^{k}\right)\right)=\operatorname{sgn}\left(x^{k}\left(I_{1}^{k}\right)\right)$, and $\operatorname{sgn}\left(c^{k}\left(I_{2}^{k}\right)\right)=\operatorname{sgn}\left(x^{k}\left(I_{2}^{k}\right)\right)$. Note that if conditions

$$
\left\{\begin{array}{l}
-A_{I_{1}}^{T}(A x-y) \in \lambda \cdot \operatorname{sgn}\left(x\left(I_{1}\right)\right)+u \cdot \operatorname{ri}\left\{\operatorname{co}_{i \in I_{1}}\left\{\operatorname{sgn}\left(x_{i}\right) \cdot w_{i} e_{i}\left(I_{1}\right)\right\}\right\}, \\
\left|A_{I_{3}}^{T}(A x-y)\right|<\lambda,
\end{array}\right.
$$

hold for an $x$, then they also hold in a small neighborhood of $x$. Taking derivative of $x$ with respect to $\lambda$ on both side of (7), we have that $-A_{I_{2}}^{T} A_{I_{2}} \frac{d x\left(I_{2}\right)}{d \lambda}=$ $\operatorname{sgn}\left(x\left(I_{2}\right)\right)$. Then there exists an interval of $\lambda$ such that the solution $x$ is a linear mapping with $\lambda$ and the optimality conditions are satisfied pointwise. The key of the algorithm is to determine the length of the interval.

The details of the homotopy algorithm are described as follows. Initially, we choose

$$
\lambda^{0}=\left\|A^{T} y\right\|_{\infty},
$$

so that $x^{0}=0$ is an optimal solution of model (1) at $\lambda:=\lambda^{0}$. At the $k$ th iteration, the algorithm updates $x^{k}$ and $\lambda^{k}$ by

$$
x^{k+1}=x^{k}+\gamma^{k} d^{k},
$$

and

$$
\lambda^{k+1}=\lambda^{k}-\gamma^{k}
$$

where $d^{k}$ is the direction along which $x^{k}$ is updated, and $\gamma^{k}$ is the step size.

1. Determine descent direction $d^{k}$.

Set $d^{k}\left(I_{1}^{k}\right)=0, d^{k}\left(I_{3}^{k}\right)=0$, and solve $d^{k}\left(I_{2}^{k}\right)$ such that the magnitudes of the coordinates of the right-hand of equation (7) decrease equally, i.e., $-A_{I_{2}^{k}}^{T}\left[A\left(x^{k}+\right.\right.$ $\left.\left.\gamma d^{k}\right)-y\right]=(\lambda-\gamma) \cdot \operatorname{sgn}\left(x^{k}\left(I_{2}^{k}\right)\right)$. Since $\operatorname{sgn}\left(x^{k}\left(I_{2}^{k}\right)\right)=\operatorname{sgn}\left(c^{k}\left(I_{2}^{k}\right)\right)$, we have

$$
A_{I_{2}^{k}}^{T} A_{I_{2}^{k}} d^{k}\left(I_{2}^{k}\right)=\operatorname{sgn}\left(c^{k}\left(I_{2}^{k}\right)\right) .
$$

2. Calculate step size $\gamma^{k}$. 
Let $x^{k}(\gamma):=x^{k}+\gamma d^{k}$ with $\gamma \geq 0$. Then the homotopy algorithm computes the step size such that $x^{k}(\gamma)$ satisfies (7) and reaches a breakpoint if either of the conditions (3), (6)-(8) is violated. There are four cases. First, an element of $-A_{I_{3}^{k}}^{T}\left(A x^{k}(\gamma)-y\right)$ increases in magnitude beyond $\lambda^{k}-\gamma$, violating (8). This occurs when

$$
\gamma_{+}^{k}=\min _{i \in I_{3}^{k}}^{+}\left\{\frac{\lambda^{k}-c_{i}^{k}}{1-a_{i}^{T} v^{k}} \mid a_{i}^{T} v^{k} \neq 1\right\} \cup\left\{\frac{\lambda^{k}+c_{i}^{k}}{1+a_{i}^{T} v^{k}} \mid a_{i}^{T} v^{k} \neq-1\right\},
$$

where $v^{k}=A d^{k}=A_{I_{2}^{k}} d^{k}\left(I_{2}^{k}\right)$, min ${ }^{+}$denotes minimum over nonnegative values and $i_{+}^{k}$ denotes the minimizing index. The second case occurs when an element of $x^{k}\left(I_{2}^{k}\right)+\gamma d^{k}\left(I_{2}^{k}\right)$ crosses zero, violating (7). This occurs when

$$
\gamma_{-}^{k}=\min ^{+}\left\{-x_{i}^{k} / d_{i}^{k} \mid i \in I_{2}^{k}, d_{i}^{k} \neq 0, \operatorname{sgn}\left(d_{i}^{k}\right)=-\operatorname{sgn}\left(c_{i}^{k}\right)\right\},
$$

where $i_{-}^{k}$ denotes the minimizing index. The third case occurs when a coordinate of $x^{k}\left(I_{2}^{k}\right)+\gamma d^{k}\left(I_{2}^{k}\right)$ breaks the box constraint $w_{i}\left|x_{i}^{k}+\gamma d_{i}^{k}\right| \leq \kappa$, violating (3). This occurs when

$$
\gamma_{--}^{k}=\min ^{+}\left\{\frac{ \pm \kappa / w_{i}-x_{i}^{k}}{d_{i}^{k}} \mid i \in I_{2}^{k}, d_{i}^{k} x_{i}^{k} \geq 0, d_{i}^{k} \neq 0, w_{i}>0\right\},
$$

where $i_{--}^{k}$ denotes the minimizing index. The fourth case occurs when the magnitude of a coordinate of $-A_{I_{1}^{k}}^{T}\left(A\left(x^{k}+\gamma d^{k}\right)-y\right)$ decreases to $\lambda^{k}-\gamma$, violating (6). This occurs when

$$
\begin{aligned}
\gamma_{++}^{k}=\min _{i \in I_{1}^{k}}^{+} \quad & \left\{\frac{\lambda^{k}-c_{i}^{k}}{1-a_{i}^{T} v^{k}} \mid c_{i}^{k}>0, a_{i}^{T} v^{k}>1\right\} \\
& \cup\left\{\frac{\lambda^{k}+c_{i}^{k}}{1+a_{i}^{T} v^{k}}, \mid c_{i}^{k}<0, a_{i}^{T} v^{k}<-1\right\},
\end{aligned}
$$

where $i_{++}^{k}$ denotes the minimizing index. In summary, $\gamma^{k}$ is determined by

$$
\gamma^{k}=\min \left\{\gamma_{+}^{k}, \gamma_{-}^{k}, \gamma_{--}^{k}, \gamma_{++}^{k}, \lambda^{k}-\lambda\right\},
$$

and index set $I^{k}, I_{1}^{k}, I_{2}^{k}$ and $I_{3}^{k}$ are updated by

$$
\begin{cases}I_{1}^{k} \stackrel{i_{++}^{k}}{\longrightarrow} I_{2}^{k}, & \text { if } \gamma^{k}=\gamma_{++}^{k}, \\ I_{1}^{k} \stackrel{i_{-}^{k}}{\longleftarrow} I_{2}^{k}, & \text { if } \gamma^{k}=\gamma_{--}^{k}, \\ I_{2}^{k} \stackrel{i_{-}^{k}}{\longrightarrow} I_{3}^{k}, I^{k+1}=I^{k} \backslash\left\{i_{-}^{k}\right\}, & \text { if } \gamma^{k}=\gamma_{-}^{k}, \\ I_{2}^{k} \stackrel{i_{+}^{k}}{\longleftarrow} I_{3}^{k}, I^{k+1}=I^{k} \cup\left\{i_{+}^{k}\right\}, & \text { if } \gamma^{k}=\gamma_{+}^{k} .\end{cases}
$$


The updated index sets are denoted by $I^{k+1}, I_{1}^{k+1}, I_{2}^{k+1}$ and $I_{3}^{k+1}$, resp. The homotopy algorithm is summarized in Algorithm 1.

Algorithm 1. homotopy Algorithm for the Box-constrained Lasso

Step 1. Set $k=0, x^{0}=0$. Initialize $\lambda^{0}$ by (9) and $I_{i}^{0}, i=1,2,3$.

Step 2. Update direction $d^{k}$ by solving equation (12).

Step 3. Determine step size $\gamma^{k}$ by solving equation (17).

Step 4. Calculate $x^{k+1}$ by equation (10), and $\lambda^{k+1}$ by equation (11). Update $I_{1}^{k}, I_{2}^{k}, I_{3}^{k}$ and $I^{k}$ by equation (18).

Step 5. Set $k:=k+1$ and go to Step 2 until $\lambda^{k}=\lambda$.

\section{Convergence Analysis}

We discuss the convergence of the algorithm under the following assumptions.

Assumption 3.1. (Unique Index Assumption). At each iteration, only one step size reaches $\gamma^{k}$ in equation (17), and the step size is made by only one index according to equation (13)-(16).

Assumption 3.2. (Non-degeneration Index Assumption). For all $k=$ $1,2, \ldots$, there does not exist an index $i \in\{1, \ldots, n\}$ satisfying

$$
\left\{\begin{array}{l}
a_{i}^{T} A d^{k}=\operatorname{sgn}\left(c_{i}^{k}\right), \\
\left|c_{i}^{k}\right|=\lambda^{k} \\
x_{i}^{k}=0 \text { or }\left|x_{i}^{k}\right|=\kappa / w_{i}, w_{i} \neq 0, \\
d_{i}^{k}=0 .
\end{array}\right.
$$

Mapping $\lambda \mapsto x^{*}(\lambda), \mathbb{R}_{+} \longrightarrow \mathbb{R}^{n}$, is called a regularization path of model (1) iff $x^{*}(\lambda)$ is an optimal solution with $\lambda>0$. For a regularization path $\lambda \mapsto x^{*}(\lambda)$, the direction of a vector $\bar{d} \in \mathbb{R}^{n}$ is called the direction of the regularization path at $x^{*}(\bar{\lambda})$ iff there exists $\bar{\gamma}>0$ such that $x^{*}(\bar{\lambda})+\gamma \bar{d}=x^{*}(\bar{\lambda}-\gamma)$ for any $\gamma \in[0, \bar{\gamma}]$.

A characterization of Condition (6) is presented in the following lemma. We omit its proof.

Lemma 3.1. Condition (6) holds if and only if

$$
\left\{\begin{array}{l}
\operatorname{sgn}\left(-A_{I_{1}}^{T}(A x-y)\right)=\operatorname{sgn}\left(x\left(I_{1}\right)\right), \\
\left|-A_{I_{1}}^{T}(A x-y)\right|_{j} \geq \lambda, \forall j .
\end{array}\right.
$$


We show that the following recursion cases do not occur: 1) an index moved out of the base $I_{2}$ enters into the base immediately at the next iteration (Recursion case I and III); 2) an index entered into the base $I_{2}$ moves out immediately at the next iteration (Recursion case II and IV).

Recursion case I: In the calculation of $\gamma_{++}^{k}$ by equation (16), there exists $i \in I_{1}^{k}$ such that $i$ moves from $I_{1}^{k}$ to $I_{2}^{k}$ and $\gamma_{++}^{k}=0$ iff

$$
\left\{\begin{array}{l}
\lambda^{k}=\left|c_{i}^{k}\right| \\
\operatorname{sgn}\left(a_{i}^{T} v^{k}\right)=\operatorname{sgn}\left(c_{i}^{k}\right) \\
\left|a_{i}^{T} v^{k}\right|>1
\end{array}\right.
$$

If index $i_{--}^{k-1}$ moves from $I_{2}^{k-1}$ to $I_{1}^{k-1}$, and $i_{--}^{k-1}$ satisfies $(25)-(27)$, then $i_{--}^{k-1}$ will move from $I_{1}^{k}$ to $I_{2}^{k}$ and recursion occurs. Theorem 1 indicates that recursion case I will not occur.

Theorem 1. At $(k-1)$ th iteration, let $i_{--}^{k-1}$ be the index that makes the step size and it moves from $I_{2}^{k-1}$ to $I_{1}^{k-1}$, then $i_{--}^{k-1}$ cannot satisfy (26) and (27) at the same time.

Similarly, it can be shown that recursion case II-IV will not occur. Theorem 2 illustrates that the homotopy algorithm generates a piecewise linear regularization path of model (1).

Theorem 2. Under the convergence assumptions, $d^{k}$ generated by the homotopy algorithm is a direction of regularization path of the box-constrained Lasso at $x^{k}$ and the sequence of $\left\{\lambda^{k}\right\}$ decreases strictly.

Proof. Along $d^{k}$, the algorithm ensures that $x:=x^{k}+\gamma d^{k}$ satisfies KKT conditions (2)-(4) with $\lambda=\lambda^{k}-\gamma$, for all $\gamma \in\left[0, \gamma^{k}\right]$. Initially, $\gamma^{0}>0$. Given that $\gamma^{k-1}>0$, we need to show $\gamma^{k}>0$.

Let $\xi_{i}^{k}=\frac{\lambda^{k}-c_{i}^{k}}{1-a_{i}^{T} v^{k}}, \quad\left(a_{i}^{T} v^{k} \neq 1\right)$ and $\eta_{i}^{k}=\frac{\lambda^{k}+c_{i}^{k}}{1+a_{i}^{T} v^{k}}, \quad\left(a_{i}^{T} v^{k} \neq-1\right)$. Define function $\zeta(t): \mathbb{R} \longrightarrow \mathbb{R}, \zeta(t)=\left\{\begin{array}{ll}t, & t \geq 0, \\ +\infty, & t<0,\end{array}\right.$ and

$$
\nu_{i}^{k}= \begin{cases}\min \left\{\zeta\left(\xi_{i}^{k}\right), \zeta\left(\eta_{i}^{k}\right)\right\}, & \text { if }\left|a_{i}^{T} v^{k}\right| \neq 1 \\ \eta_{i}^{k}, & \text { if } a_{i}^{T} v^{k}=1, \operatorname{sgn}\left(c_{i}^{k-1}\right)=-1 \\ \xi_{i}^{k}, & \text { if } a_{i}^{T} v^{k}=-1, \operatorname{sgn}\left(c_{i}^{k-1}\right)=1\end{cases}
$$

then we have by (13) that $\gamma_{+}^{k}=\min _{i \in I_{3}^{k}} \nu_{i}^{k}$. Moreover, for any $i \in\{1, \ldots, n\}$, 
we have by (11) and $x^{k}=x^{k-1}+\gamma^{k-1} d^{k-1}$ that

$$
\left\{\begin{array}{l}
c_{i}^{k-1}-\gamma^{k-1} a_{i}^{T} v^{k-1}=c_{i}^{k} \\
\lambda^{k}=\lambda^{k-1}-\gamma^{k-1}
\end{array}\right.
$$

Denote $i^{k-1}$ the index that moves in or out of the set $I_{2}^{k-1}$ at $(k-1)$ th iteration. It can be shown that $\gamma_{+}^{k}>0, \gamma_{-}^{k}>0, \gamma_{++}^{k}>0$ and $\gamma_{--}^{k}>0$. We prove $\gamma_{++}^{k}>0$, the other step sizes follow similar arguments.

Suppose for contrary that $\gamma_{++}^{k}=0$, which occurs if and only if there exists $i \in I_{1}^{k}$ such that (25)-(27) hold. By Theorem 1, we have $i^{k-1}$, does not satisfy (25)-(27) simultaneously, if $i^{k-1} \in I_{1}^{k}$. Hence, by Assumption 3.1, $i \in I_{1}^{k} \backslash$ $\left\{i^{k-1}\right\} \subseteq I_{1}^{k-1}$. Together with Lemma 3.1 and $\operatorname{sgn}\left(x_{i}^{k-1}\right)=\operatorname{sgn}\left(x_{i}^{k}\right)=\operatorname{sgn}\left(c_{i}^{k}\right)$, we have

$$
\left\{\begin{array}{l}
\left|c_{i}^{k-1}\right| \geq \lambda^{k-1}, \\
\operatorname{sgn}\left(c_{i}^{k-1}\right)=\operatorname{sgn}\left(x_{i}^{k-1}\right)=\operatorname{sgn}\left(c_{i}^{k}\right) .
\end{array}\right.
$$

It follows by (25), (29), (30) and $\gamma^{k-1}>0$ that $\left|c_{i}^{k-1}\right| \geq \lambda^{k-1}=\lambda^{k}+\gamma^{k-1}>$ $\lambda^{k}=\left|c_{i}^{k}\right|$. Combining with (28) and (31) we have

$$
\operatorname{sgn}\left(c_{i}^{k-1}\right)=\operatorname{sgn}\left(a_{i}^{T} v^{k-1}\right)
$$

By (25), (28) and (29) we have

$$
\left|c_{i}^{k-1}-\gamma^{k-1} a_{i}^{T} v^{k-1}\right|=\lambda^{k-1}-\gamma^{k-1} .
$$

According to (28), (30), (32) and (33), we have that if $c_{i}^{k}>0$, then $a_{i}^{T} v^{k-1} \geq 1$. Note that the case $a_{i}^{T} v^{k-1}=1, c_{i}^{k-1}=\lambda^{k-1}$ does not occur; otherwise, it violates Assumption 3.2 according to $\left|x_{i}^{k-1}\right|=\kappa / w_{i}, d_{i}^{k-1}=0$ (because $i \in I_{1}^{k}$ ). Hence, $a_{i}^{T} v^{k-1}>1$. Then, (33) implies that $\gamma^{k-1}=\frac{\lambda^{k-1}-c_{i}^{k-1}}{1-a_{i}^{T} v^{k-1}}, a_{i}^{T} v^{k-1}>1$. Similarly, we have that if $c_{i}^{k-1}<0, \gamma^{k-1}=\frac{\lambda^{k-1}+c_{i}^{k-1}}{1+a_{i}^{T} v^{k-1}}, a_{i}^{T} v^{k-1}<-1$. Then, according to (16) and Assumption 3.1, $i$ makes the step size of $\gamma^{k-1}$ and should move from $I_{1}^{k-1}$ to $I_{2}^{k-1}$ at $(k-1)$ th iteration, which contradicts the fact that $i \neq i^{k-1}$ and $i \in I_{1}^{k}$.

By equation (17), we have $\gamma^{k}>0$, which concludes the proof. 


\section{Concluding Remarks}

Convergence assumptions are necessary in the algorithm implementation; otherwise, degeneration may occur. For an instance of $\lambda^{k}=\lambda^{k+1}=\ldots=\lambda^{k+q}$ with $q>0$, if the index sets and the value of $\lambda$ at iteration $k+q$ turns out to be the same as those at iteration $k$, then the algorithm will fall into infinite loops. In this work, we presented mild convergence assumptions and proved that the algorithm generated the entire regularization path in the procedure of iterations.

\section{Acknowledgements}

This research is partially supported by Natural Science Foundation of Shandong Province under Grant ZR2014AP004 and Fundamental Research Funds for the Central Universities under Grant 15CX02051A.

\section{References}

[1] R. Tibshirani, Regression shrinkage and selection via the lasso, J. Roy. Stat. Soc.: Series $B, 58$ (1996), 267-288.

[2] N. Meinshausen, G. Rocha, and B. Yu, Discussion: A tale of three cousins: Lasso, L2Boosting and Dantzig, Ann. Stat., 35 (2007), 2373-2384.

[3] M. R. Osborne, B. Presnell, and B. A. Turlach, A new approach to variable selection in least squares problems, IMA J. Numer. Anal., 20 (2000), 389-403.

[4] B. Efron, T. Hastie, I. Johnstone, et al. Least angle regression, Ann. Stat., 32 (2004), 407-499.

[5] F. Bach, R. Jenatton, and J. Mairal, et al. Optimization with sparsity-inducing penalties, Found. Trends Mach. Learn., 4 (2011), 1-108.

[6] D. L. Donoho, and Y. Tsaig, Fast solution of 11-norm minimization problems when the solution may be sparse, IEEE Trans. Inform. Theory, 54 (2008), 4789-4812.

[7] X. J. Liang, Z. H. Xia, L. P. Pang, et al. Measure prediction capability of data for collaborative filtering. Knowledge and Information Systems. (2016), 1-30. 